\title{
Rare decays of flavoured mesons at the LHC
}

\author{
Albert Puig* \\ Physik-Institut, Universität Zürich (Switzerland) \\ E-mail: albert.puigecern.ch
}

In absence of strong, direct signs of New Physics at the LHC, rare decays of heavy flavoured hadrons constitute an ideal laboratory for indirectly exploring energies beyond those of the LHC in order to look for deviations from the Standard Model. The main results regarding flavour changing neutral current transitions obtained at the LHC are presented here, with particular emphasis put on $b \rightarrow s$ transitions, in which tensions with the Standard Model have been observed.

Fourth Annual Large Hadron Collider Physics

13-18 June 2016

Lund, Sweden

${ }^{*}$ Speaker. 


\section{Introduction}

Quarks and leptons in the Standard Model (SM) of particle physics are organized in flavours. Changes between flavours can only occur through the charged current weak interaction-that is, mediated by a $W$ boson - and transitions between same-charge fermions must occur through second order, loop processes [1]. Since the Cabibbo-Kobayashi-Maskawa (CKM) quark mixing matrix [2,3], which governs transitions between flavours, is found to be approximately diagonal, generation-changing processes are suppressed. As a consequence, processes involving flavour changing neutral currents (FCNC) are predicted to be rare within the SM.

Observables related to these decays - branching fractions, $C P$ asymmetries, kinematic distributions, among others — can be predicted in the SM with low theoretical uncertainty. Many models of New Physics (NP) predict noticeable differences in the measured quantities, making the study of rare decays of flavoured hadrons an ideal laboratory for studying physics Beyond the Standard Model. In particular, loop-mediated processes allow indirect access to quantum corrections from degrees of freedom at larger scales and provide excellent complementarity to direct searches of new phenomena.

FCNC transitions with $|\Delta B|=|\Delta S|=1$ are described by a low energy effective field theory in the form of an Operator Product Expansion [4-6]:

$$
\mathscr{H}_{\mathrm{eff}}=-\frac{4 G_{F}}{\sqrt{2}} V_{t b} V_{t s}^{*} \frac{\alpha}{4 \pi} \sum_{i}\left\{C_{i} O_{i}+C_{i}^{\prime} O_{i}^{\prime}\right\},
$$

where $G_{F}$ is the Fermi constant, $V_{i j}$ are CKM matrix elements and $\alpha_{e}$ is the fine structure constant. The $O_{i}^{(\prime)}$ local operators take into account all possible left(right)-handed Lorentz structures and come with their corresponding Wilson coefficients $C_{i}^{(\prime)}$. The fact that the charged current interaction is left-handed implies that the Wilson coefficients corresponding to the right-handed $O_{i}^{\prime}$ operators are suppressed by $\mathscr{O}\left(m_{s} / m_{b}\right)$.

The most important operators for the study of rare $b \rightarrow s \gamma, b \rightarrow s \ell^{+} \ell^{-}$and $b \rightarrow \ell^{+} \ell^{-}$decays ${ }^{1}$ are

$$
\begin{aligned}
O_{7}^{(\prime)} & =\frac{m_{b}}{e} \bar{s} \sigma^{\mu v} P_{R(L)} b F_{\mu v}, \\
O_{9}^{(\prime)} & =\bar{s} \gamma_{\mu} P_{R(L)} b \bar{\ell} \gamma^{\mu} \ell, \\
O_{10}^{(\prime)} & =\bar{s} \gamma_{\mu} P_{R(L)} b \bar{\ell} \gamma^{\mu} \gamma_{5} \ell, \\
O_{S}^{(\prime)} & =\bar{s} P_{R(L)} b \bar{\ell} \ell \\
O_{P}^{(\prime)} & =\bar{s} P_{R(L)} b \bar{\ell} \gamma_{5} \ell
\end{aligned}
$$

where $P_{L(R)}$ denotes the left(right)-handed chiral projector and $F_{\mu \nu}$ the electromagnetic field strength tensor. Radiative $b \rightarrow s \gamma$ transitions are controlled by the photon penguin operator $O_{7}^{(\prime)}$; semileptonic $b \rightarrow s \ell^{+} \ell^{-}$processes receive contributions from $O_{7}^{(\prime)}$ and the electroweak penguin operators $O_{9}^{(\prime)}$ and $O_{10}^{(\prime)}$; and the fully leptonic $b \rightarrow \ell^{+} \ell^{-}$decays are ruled by $O_{9,10}^{(\prime)}$ and the scalar and pseudoscalar penguin operators $O_{S}^{(\prime)}$ and $O_{P}^{(\prime)}$.

\footnotetext{
${ }^{1} b \rightarrow d \gamma$ and $b \rightarrow d \ell^{+} \ell^{-}$transitions are treated analogously, but are more suppressed due to the replacement of $V_{t b} V_{t s}^{*}$ by $V_{t b} V_{t d}^{*}$ in Eq. 1.1 .
} 

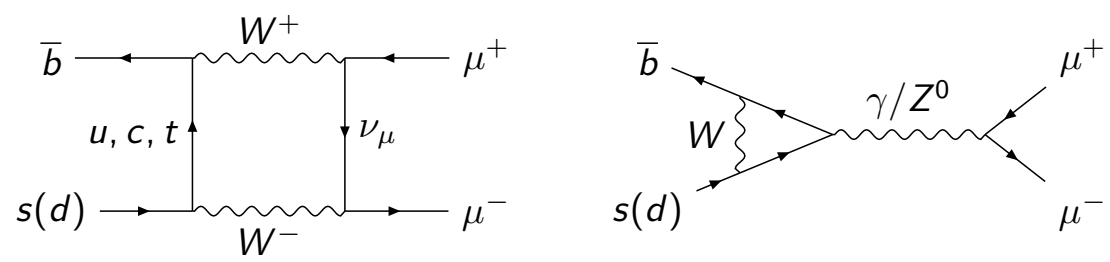

Figure 1: Feynman diagrams of the $B_{s}^{0} \rightarrow \mu^{+} \mu^{-}$and $B^{0} \rightarrow \mu^{+} \mu^{-}$decays.

Effects from NP can be easily incorporated in Eq. 1.1 by adding an extra term for each operator

$$
\mathscr{H}_{\mathrm{eff}}^{\mathrm{NP}}=\sum_{i} \frac{C_{i}^{\mathrm{NP}}}{\Lambda_{\mathrm{NP}}^{2}} O_{i}^{\mathrm{NP}}
$$

where $\Lambda_{\mathrm{NP}}$ is the NP scale.

Measurements of different observables and decay modes can then be combined in global fits of Wilson coefficients and used to constrain NP contributions in FCNC. Hence, the strategy in the indirect searches for NP in rare decays is to perform many measurements, study their discrepancies and agreements with the SM through global fits, and try to solve the puzzle: how do we explain all these results in a single model, i.e., which is the structure of the model beyond the SM?

The current situation in terms of rare decay results and the constraints they impose on NP are discussed in the next sections.

\section{Fully leptonic $b \rightarrow \ell^{+} \ell^{-}$decays}

The dileptonic $B_{s}^{0} \rightarrow \mu^{+} \mu^{-}$and $B^{0} \rightarrow \mu^{+} \mu^{-}$decays are suppressed due to their loop only diagrams, the involved CKM matrix elements and the particular helicity structure of a pseudoscalar decaying into a pair of leptons (Fig. 1), and thus are very rare in the SM. More precisely, the time-integrated branching fractions are predicted to be [7]

$$
\begin{aligned}
& \mathscr{B}\left(B_{s}^{0} \rightarrow \mu^{+} \mu^{-}\right)=(3.66 \pm 0.23) \times 10^{-9}, \\
& \mathscr{B}\left(B^{0} \rightarrow \mu^{+} \mu^{-}\right)=(1.06 \pm 0.09) \times 10^{-10},
\end{aligned}
$$

where the main uncertainties come from the knowledge of the decay constants and the CKM matrix elements. Several NP models including sizeable scalar or pseudoscalar operators can enhance the branching fractions of one or both the $B_{s}^{0}$ and the $B^{0}$ modes [8], as shown in Fig. 2.

Culminating a story started more than thirty years ago by the CLEO collaboration [9], the LHCb and CMS collaborations performed a combined analysis of the data collected during Run I, and reported the first observation of $B_{s}^{0} \rightarrow \mu^{+} \mu^{-}$with a significance of $6.2 \sigma$ and an evidence for $B^{0} \rightarrow \mu^{+} \mu^{-}$at $3 \sigma$. The measured branching fractions [10]

$$
\begin{aligned}
\mathscr{B}\left(B_{s}^{0} \rightarrow \mu^{+} \mu^{-}\right) & =\left(2.8_{-0.6}^{+0.7}\right) \times 10^{-9}, \\
\mathscr{B}\left(B^{0} \rightarrow \mu^{+} \mu^{-}\right) & =\left(3.9_{-1.4}^{+1.6}\right) \times 10^{-10},
\end{aligned}
$$

are compatible with the SM at $1.2 \sigma$ and $2.2 \sigma$, respectively, as shown in Fig. 3. Despite this fact, these results are very important as they put strong constraints on NP scenarios [11]. 


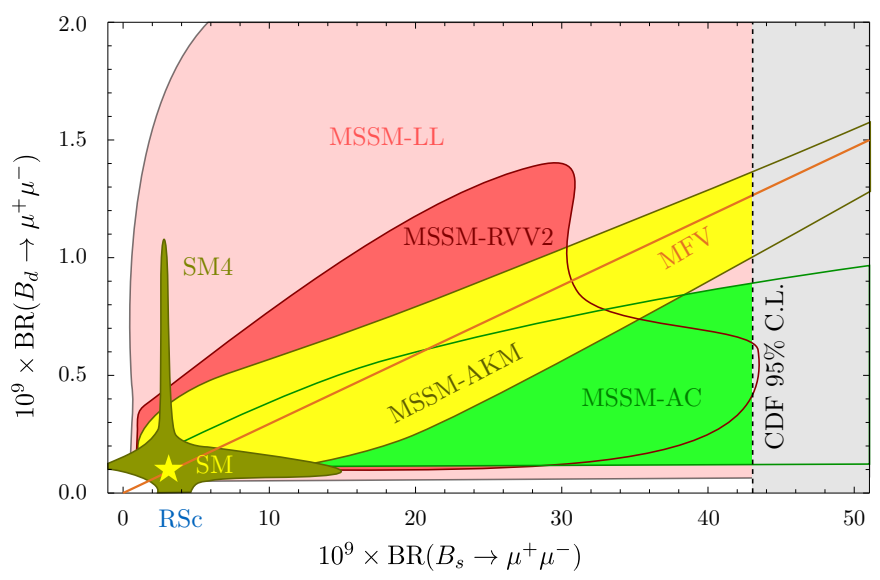

Figure 2: Correlation between the branching fractions of $B_{s}^{0} \rightarrow \mu^{+} \mu^{-}$and $B^{0} \rightarrow \mu^{+} \mu^{-}$in several NP models [8]. The grey area was the one excluded experimentally before the LHC.
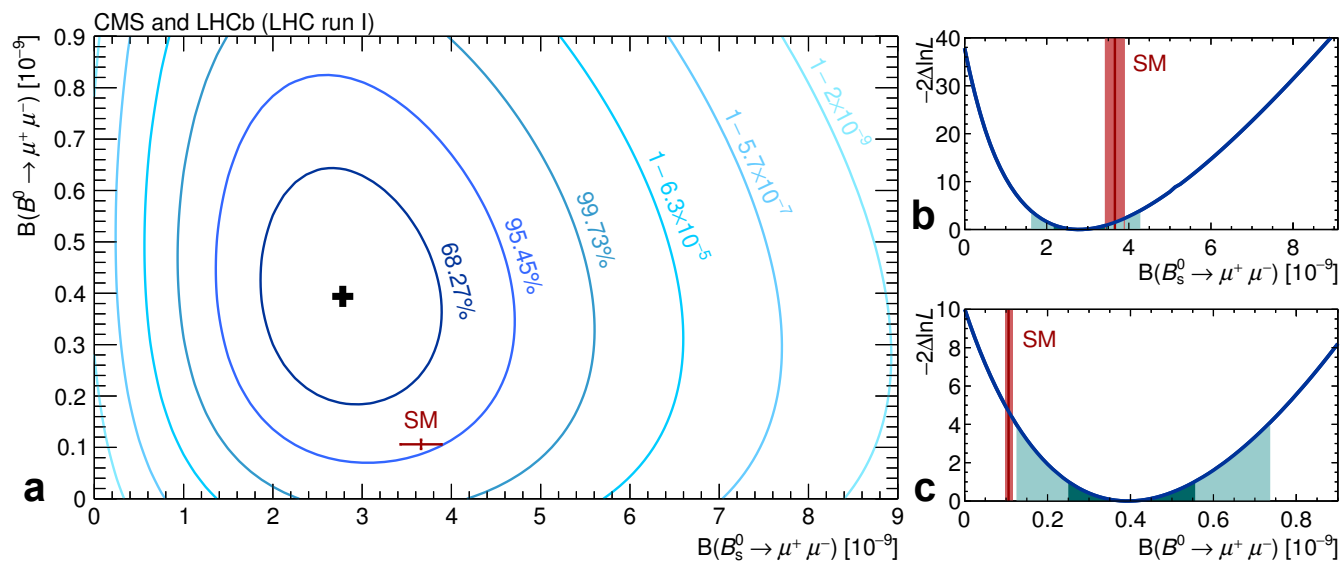

Figure 3: Likelihood contours in the $\mathscr{B}\left(B_{s}^{0} \rightarrow \mu^{+} \mu^{-}\right)$vs $\mathscr{B}\left(B^{0} \rightarrow \mu^{+} \mu^{-}\right)$plane (a), with variations of the $-2 \Delta \ln \mathscr{L}$ test statistic for each of the modes shown in (b) and (c) [10].

The ATLAS collaboration has also found compatible results with CMS and LHCb [12]:

$$
\begin{aligned}
& \mathscr{B}\left(B_{s}^{0} \rightarrow \mu^{+} \mu^{-}\right)=\left(0.9_{-0.8}^{+1.1}\right) \times 10^{-9}, \\
& \mathscr{B}\left(B^{0} \rightarrow \mu^{+} \mu^{-}\right)<4.2 \times 10^{-10} \text { at } 95 \% \text { C.L.. }
\end{aligned}
$$

\section{Semileptonic $b \rightarrow s(d) \ell^{+} \ell^{-}$decays}

Semileptonic $b \rightarrow s(d) \ell^{+} \ell^{-}$decays have been extensively studied at the LHC, where the signal yields of many modes are large enough for precision measurements. Results on differential branching fractions and angular distributions, as well as ratios between muonic and electronic decays, have provided many constraints on NP and have yielded interesting tensions with the SM, and will be discussed in the following.

Differential branching fractions The LHC measurements of the branching fractions of $B \rightarrow$ $K \mu^{+} \mu^{-}$[13], $B \rightarrow K^{*} \mu^{+} \mu^{-}$[13-16], $B_{s}^{0} \rightarrow \phi \mu^{+} \mu^{-}$[17] and $\Lambda_{b}^{0} \rightarrow \Lambda \mu^{+} \mu^{-}$[18], performed in 

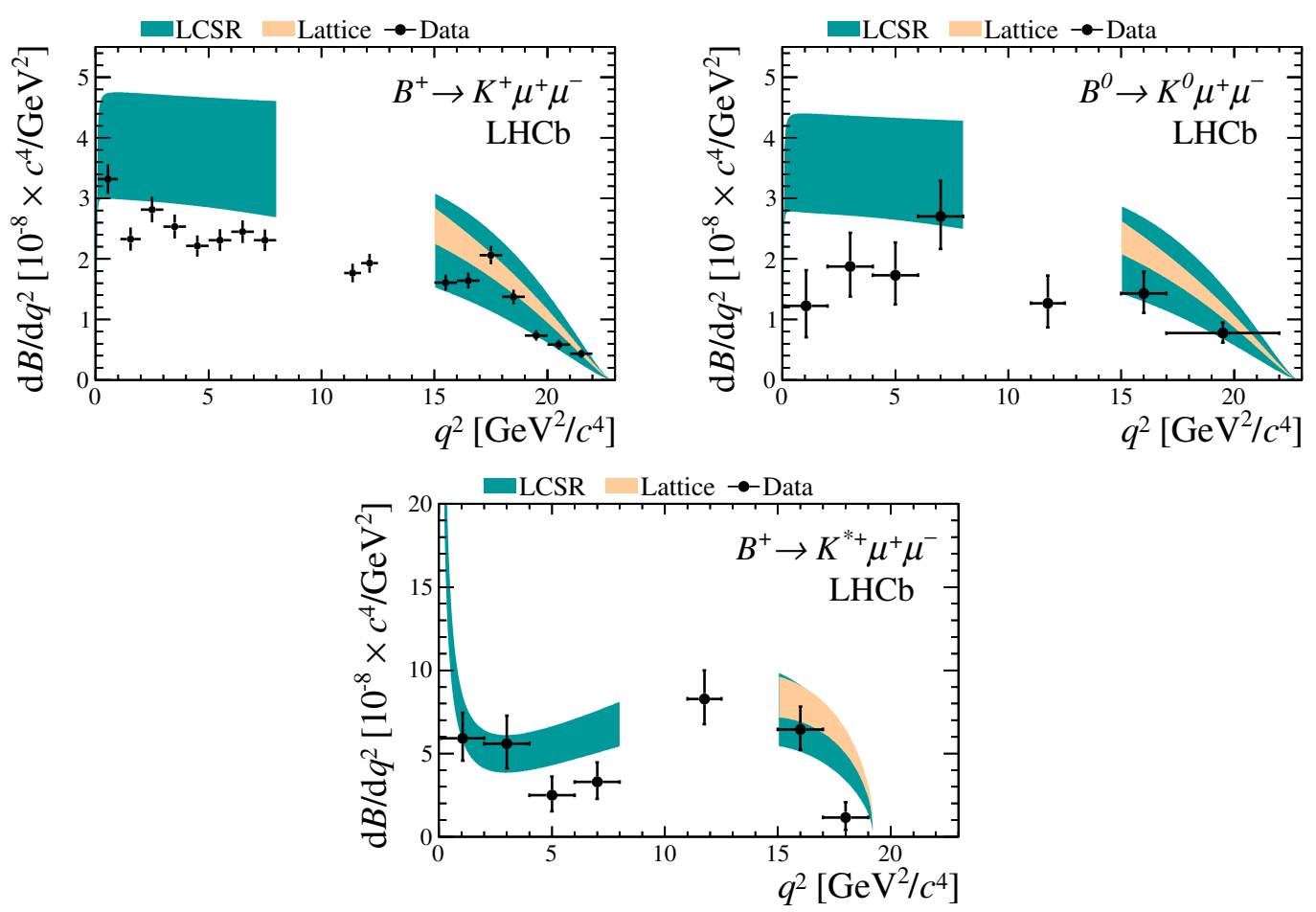

Figure 4: Differential branching fraction in bins of $q^{2}$ of the $B^{+} \rightarrow K^{+} \mu^{+} \mu^{-}$(top left), $B^{0} \rightarrow K^{0} \mu^{+} \mu^{-}$(top right) and $B^{+} \rightarrow K^{*+} \mu^{+} \mu^{-}$(bottom) decays as measured by LHCb [13]. Theoretical predictions obtained with LCSR [19] and lattice QCD [20,21] calculations are shown for comparison.

bins of the dilepton mass squared $\left(q^{2}\right)$, are much more precise than the corresponding theoretical predictions, sensitive to hadronic uncertainties in the form factors. These theoretical uncertainties, typically of the order of $30 \%$, limit the sensitivity to NP, but are expected to improve in the future with progress from lattice QCD.

While some precision results from the large datasets collected by LHCb point towards lower values than the SM prediction in some cases, as shown in Figs. 4 and 5, the measurements are in general compatible with the SM prediction. The branching fractions of the $B^{0} \rightarrow K^{* 0} \mu^{+} \mu^{-}$, measured both by CMS [15,16] and LHCb [14], and the $\Lambda_{b}^{0} \rightarrow \Lambda \mu^{+} \mu^{-}$decays, affected by large form factor uncertainties, don't show any deviations from the SM prediction, as shown in Figs. 6 and 7. The latest LHCb result on $B^{0} \rightarrow K^{* 0} \mu^{+} \mu^{-}$[14] is the first one to include a measurement of the $S$-wave component in the $K^{+} \pi^{-}$system, in contrast with previous studies, which considered it small and treated it as a systematic uncertainty. As the theory predictions are made for purely resonant $P$-wave, an accurate assessment of the $S$-wave fraction is critical, and, as can be seen on the left plot in Fig. 7, agreement between the measurement and the SM prediction from lattice QCD is good.

As a complement to the measurement of $b \rightarrow s \ell^{+} \ell^{-}$transitions, $b \rightarrow d \ell^{+} \ell^{-}$decays, suppressed by $\left|V_{t d} / V_{t s}\right|^{2}$, allow to test whether NP-if any-is minimally flavour violating (MFV). The LHCb collaboration has the $B^{+} \rightarrow \pi^{+} \mu^{+} \mu^{-}$[26] and has found good compatibility with the SM predictions, as can be seen in Fig. 8, with the uncertainty in the result still dominated by statistics. Further improvements in this study, as well as observations of more $b \rightarrow d \ell^{+} \ell^{-}$modes, are expected in the 


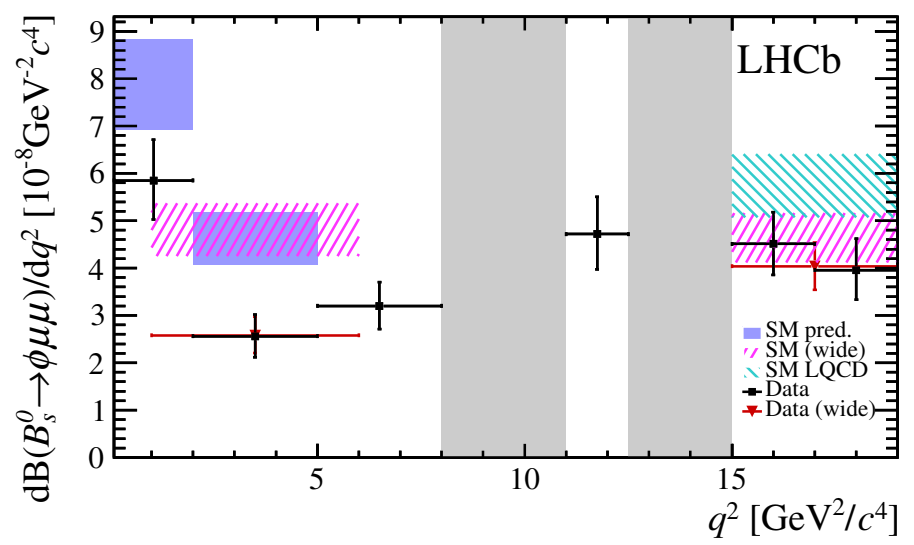

Figure 5: Differential branching fraction in bins of $q^{2}$ of the $B_{s}^{0} \rightarrow \phi \mu^{+} \mu^{-}$decay as measured by LHCb [17]. The SM prediction with LCSR [22,23] is overlaid in purple and magenta for different $q^{2}$ binning schemes, while the LQCD prediction for high- $q^{2}$ is showed in cyan [21].

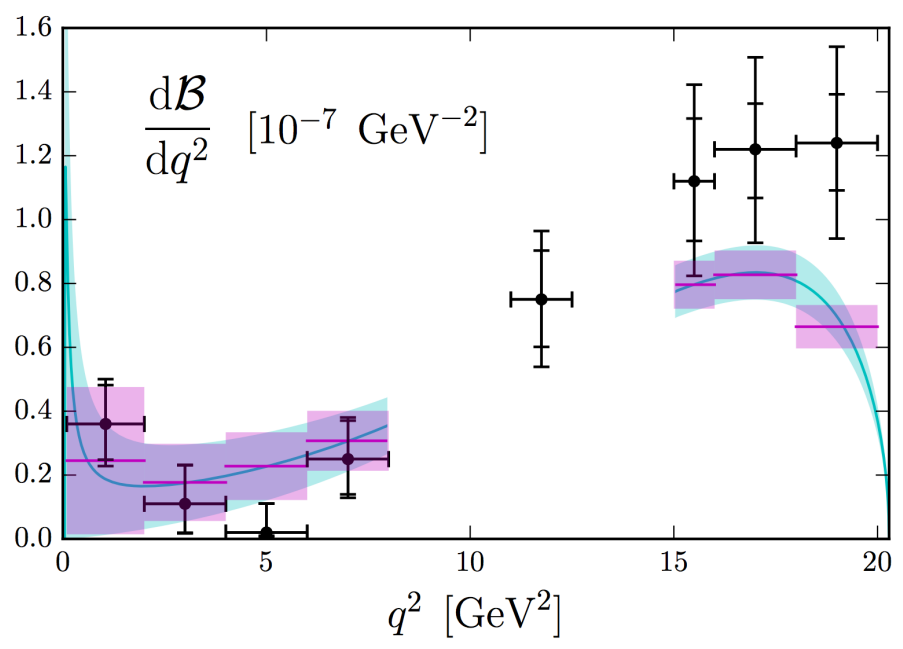

Figure 6: Differential branching fraction in bins of $q^{2}$ of the $\Lambda_{b}^{0} \rightarrow \Lambda \mu^{+} \mu^{-}$decay as measured by LHCb [18]. The plot, obtained from Ref. [24], shows an updated SM prediction from lattice QCD with respect to that included in Ref. [18].

coming years.

Angular distributions The angular distributions of $b \rightarrow s \ell^{+} \ell^{-}$decays provide a large number of observables with different sensitivities to different types of NP. In particular, each of the observables arising from the angular distributions_-or combinations thereof_-has a different dependence on the Wilson coefficients-mainly $C_{7}^{(\prime)}, C_{9}^{(\prime)}$ and $C_{10}^{(\prime)}$ - and form factors.

The $B^{0} \rightarrow K^{* 0} \mu^{+} \mu^{-}$angular distribution depends on three angles: the direction of the $\mu^{+}\left(\mu^{-}\right)$ with respect to the $B^{0}\left(\bar{B}^{0}\right)$ in the dimuon rest frame $\left(\theta_{l}\right)$, the direction of the kaon with respect to the $B$ in the $K \pi$ system rest frame $\left(\theta_{K}\right)$, and the angle between the dimuon plane and the $K \pi$ system 

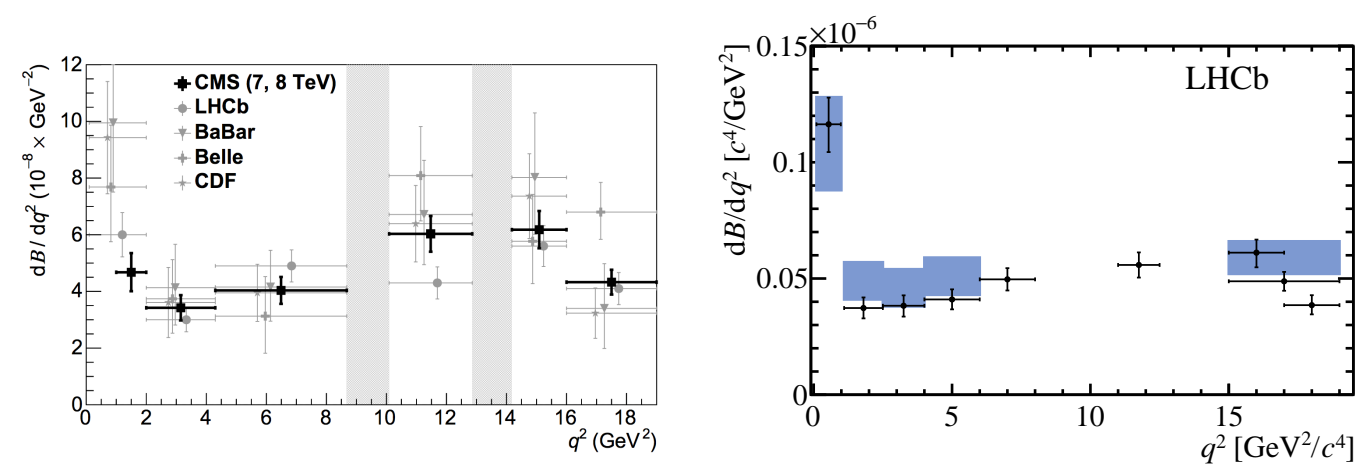

Figure 7: On the left, differential branching fraction in bins of $q^{2}$ of the $B^{0} \rightarrow K^{* 0} \mu^{+} \mu^{-}$as measured by $\mathrm{CMS}$, LHCb, the $B$ factories and CDF, taken from Ref. [16]. On the right, latest differential branching fraction LHCb result in bins of $q^{2}$ of the purely resonant $B^{0} \rightarrow K^{* 0} \mu^{+} \mu^{-}$decay, after measuring the $S$-wave component [14]; the overlaid theory prediction is from Refs. [23, 25].

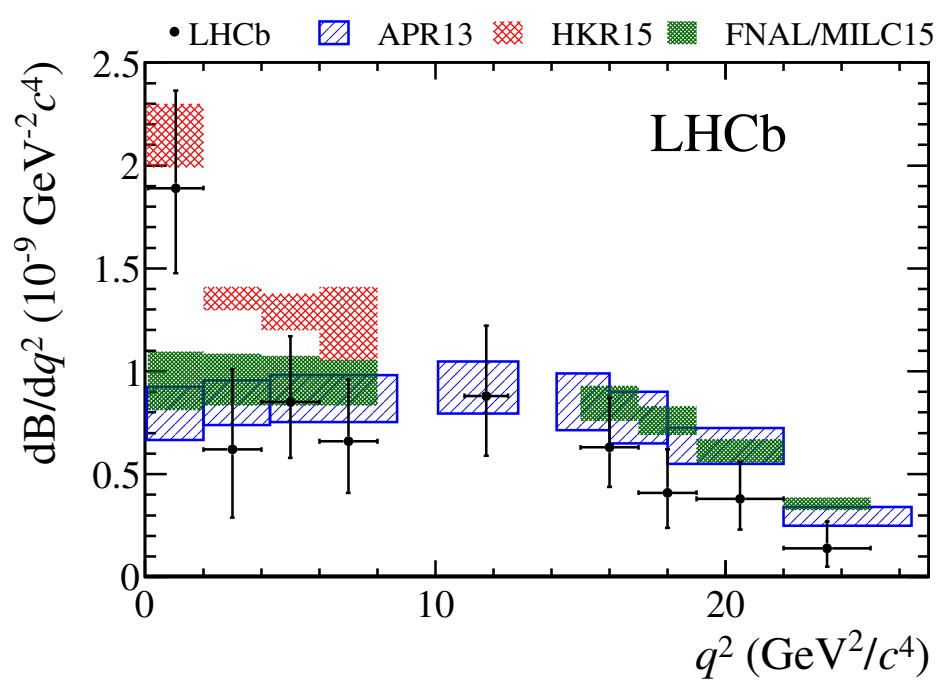

Figure 8: Differential branching fraction in bins of $q^{2}$ of the $B^{+} \rightarrow \pi^{+} \mu^{+} \mu^{-}$decay as measured by LHCb [26], compared with the APR13 [27], HKR15 [28] and lattice QCD FNAL/MILC15 [29] SM predictions.

plane $(\phi)$. The $C P$ averaged differential decay rate in terms of these angles and $q^{2}$ can be written as:

$$
\begin{aligned}
\frac{1}{\mathrm{~d}(\Gamma+\bar{\Gamma}) / \mathrm{d} q^{2}} \frac{\mathrm{d}^{4}(\Gamma+\bar{\Gamma})}{\mathrm{d} q^{2} \mathrm{~d} \vec{\Omega}}=\frac{9}{32 \pi} & {\left[\frac{3}{4}\left(1-F_{\mathrm{L}}\right) \sin ^{2} \theta_{K}+F_{\mathrm{L}} \cos ^{2} \theta_{K}\right.} \\
& +\frac{1}{4}\left(1-F_{\mathrm{L}}\right) \sin ^{2} \theta_{K} \cos 2 \theta_{\ell} \\
& -F_{\mathrm{L}} \cos ^{2} \theta_{K} \cos 2 \theta_{\ell}+S_{3} \sin ^{2} \theta_{K} \sin ^{2} \theta_{\ell} \cos 2 \varphi \\
& +S_{4} \sin 2 \theta_{K} \sin 2 \theta_{\ell} \cos \varphi+S_{5} \sin 2 \theta_{K} \sin \theta_{\ell} \cos \varphi \\
& +\frac{4}{3} A_{\mathrm{FB}} \sin ^{2} \theta_{K} \cos \theta_{\ell}+S_{7} \sin 2 \theta_{K} \sin \theta_{\ell} \sin \varphi \\
& \left.+S_{8} \sin 2 \theta_{K} \sin 2 \theta_{\ell} \sin \varphi+S_{9} \sin ^{2} \theta_{K} \sin ^{2} \theta_{\ell} \sin 2 \varphi\right],
\end{aligned}
$$

where $S_{i}, F_{\mathrm{L}}$ (fraction of longitudinal polarization of the $K^{* 0}$ ) and $A_{\mathrm{FB}}$ (forward-backward asymmetry 

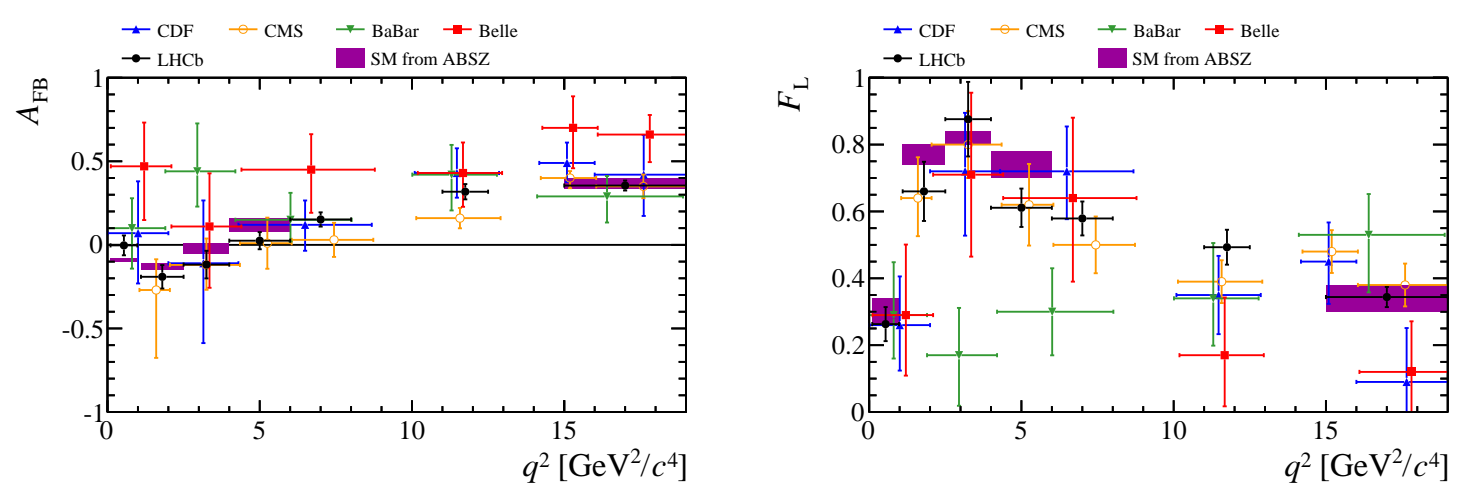

Figure 9: Forward-backward asymmetry of the dimuon system, $A_{\mathrm{FB}}$, (left) and fraction of longitudinal polarization of the $K^{* 0}$ mesons, $F_{\mathrm{L}}$, (right) in $B^{0} \rightarrow K^{* 0} \mu^{+} \mu^{-}$decays in bins of $q^{2}$ as measured by the CDF, $\mathrm{BaBar}$, Belle, CMS and LHCb collaborations, taken from Ref. [34]. The SM prediction is obtained from Ref. [23].

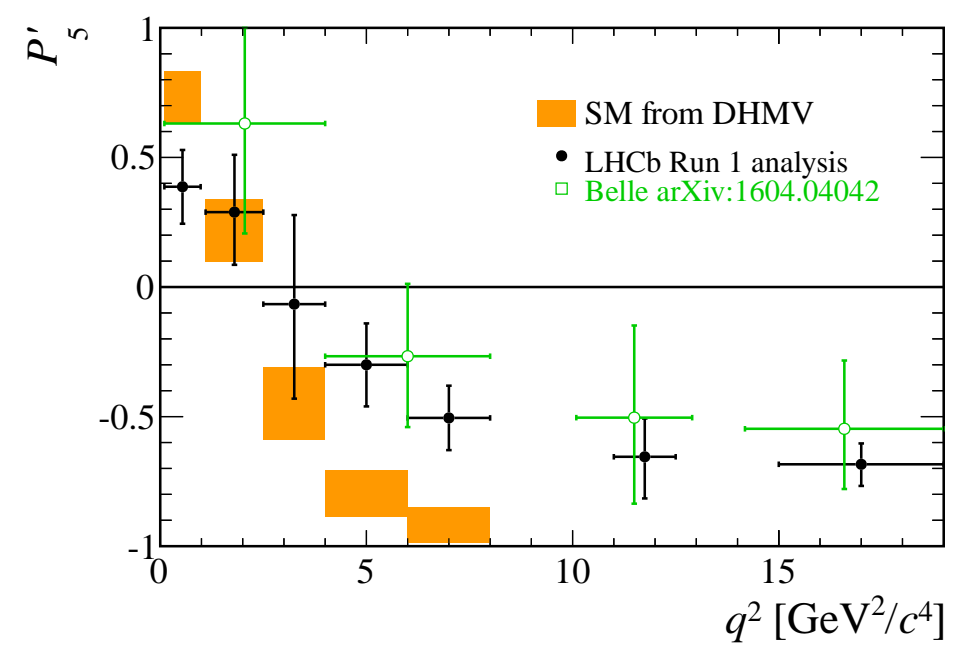

Figure 10: The optimized angular observable $P_{5}^{\prime}$ in bins of $q^{2}$ as measured by LHCb and Belle, overlaid with the SM prediction from Ref. [36].

of the dilepton system) are the observables to be measured. It is possible to build theoretically cleaner observables by combining helicity amplitudes to exploit cancellations. In particular, the $P_{i}^{\prime}$ set of observables [30], such as

$$
P_{5}^{\prime}=\frac{S_{5}}{\sqrt{F_{\mathrm{L}}\left(1-F_{\mathrm{L}}\right)}}
$$

focuses on reducing the dependence on form factors, thus reducing the theoretical uncertainty.

While the measurements of the standard $B^{0} \rightarrow K^{* 0} \mu^{+} \mu^{-}$angular observables performed by CDF [31], BaBar [32], Belle [33], CMS [16] and LHCb [34] have been found to be compatible with the SM (see Fig. 9), the optimized $P_{5}^{\prime}$ observable, measured by LHCb and Belle [35], presents a large local discrepancy between data and the SM prediction, shown in Fig. 10, at the level of 3.7 $\sigma$.

Measurements of simplified angular distributions in bins of $q^{2}$ carried out by LHCb in the $B_{s}^{0} \rightarrow \phi \mu^{+} \mu^{-}$[17] and $\Lambda_{b}^{0} \rightarrow \Lambda \mu^{+} \mu^{-}$[18] decays don't show large deviations from the SM, and 

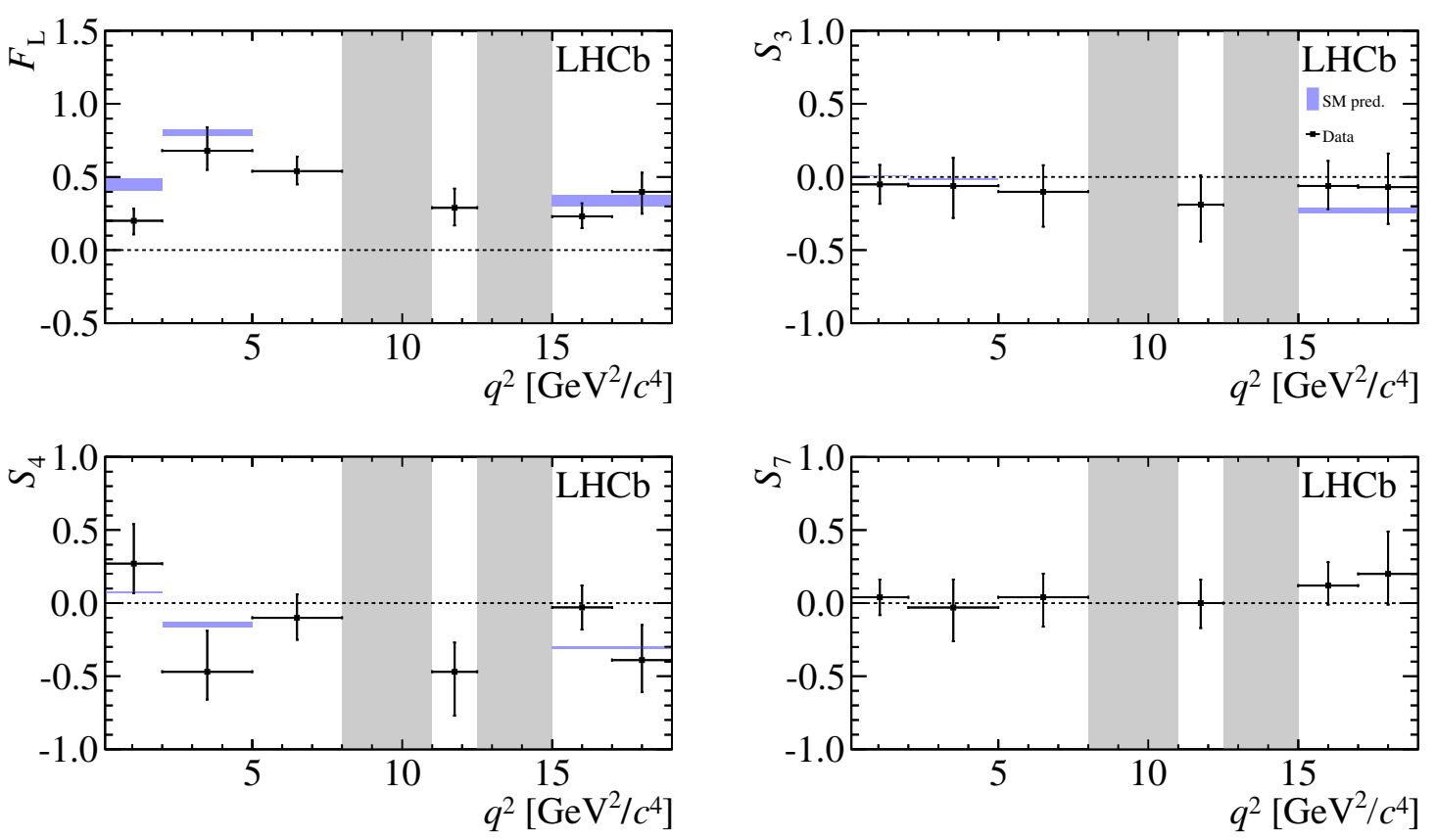

Figure 11: Fraction of longitudinal polarization of the $K^{* 0}$ mesons, $F_{\mathrm{L}}, S_{3}, S_{4}$ and $S_{9}$ distributions in $B_{s}^{0} \rightarrow \phi \mu^{+} \mu^{-}$decays in bins of $q^{2}$ as measured by LHCb [17]. The SM prediction is obtained from Refs. [22,23].
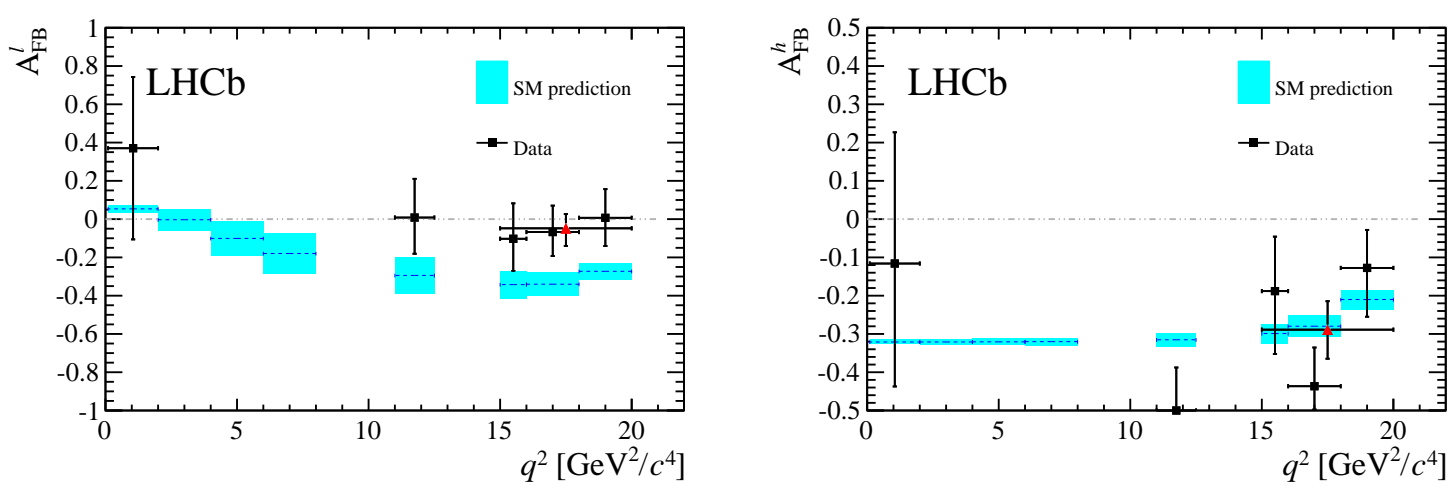

Figure 12: Forward-backward asymmetry of the dimuon system, $A_{\mathrm{FB}}^{l}$, (left) and forward-backward asymmetry of the hadron system, $A_{\mathrm{FB}}^{h}$, (right) in $\Lambda_{b}^{0} \rightarrow \Lambda \mu^{+} \mu^{-}$decays in bins of $q^{2}$ as measured by LHCb [18]. The SM prediction is obtained from Ref. [37].

can be seen in Figs. 11 and 12, respectively.

Additionally, the LHCb collaboration has studied the angular distribution of the $B^{0} \rightarrow K^{* 0} e^{+} e^{-}$ decay in the low $-q^{2}$ region [38], performing some angular transformations to reduce the number of 
angular observables on account for the limited signal yield. The obtained results,

$$
\begin{aligned}
F_{\mathrm{L}} & =0.16 \pm 0.06 \pm 0.03 \\
A_{T}^{\mathrm{Re}} & =0.10 \pm 0.18 \pm 0.05 \\
A_{T}^{(2)} & =-0.23 \pm 0.23 \pm 0.05 \\
A_{T}^{\mathrm{Im}} & =0.14 \pm 0.22 \pm 0.05
\end{aligned}
$$

are compatible with the SM predictions $[39,40]$ and help constrain the $C_{7}^{(\prime)}$ Wilson coefficient thanks to the low lepton mass.

Global fits With the wealth of measurements produced at the LHC, including some tensions with the SM predictions, it becomes possible to gain insight on possible NP contributions to the Wilson coefficients through the combination of these measurements, i.e., performing global fits of $b \rightarrow s$ observables. Taking into account more than eighty observables from $b \rightarrow \ell^{+} \ell^{-}, b \rightarrow s(d) \ell^{+} \ell^{-}$and $b \rightarrow s \gamma$ transitions, measured by six experiments, most global fits [41-44] prefer a negative NP contribution $C_{9} \sim-1$, with other NP parameters consistent with zero, as shown in Fig. 13. While this destructive contribution would better accommodate the data by reducing the branching fraction of $b \rightarrow s(d) \mu^{+} \mu^{-}$decays and modifing the angular distribution of $B^{0} \rightarrow K^{* 0} \mu^{+} \mu^{-}$to be more consistent with the $P_{5}^{\prime}$ measurements, it is worth noting that these fits are still compatible with the SM prediction at $3-4.6 \sigma$.

Further measurements $b \rightarrow s \ell^{+} \ell^{-}$transitions, in particular of angular observables, with the LHC Run II dataset, will be crucial in the clarification of the real nature of this tension-currently one of the most significant in flavour physics.

Lepton flavour universality Another interesting tension with the SM, complementary to those described above, arises from the studies of lepton flavour universality. In the SM, with the exception of the Higgs boson, particles couple equally to different lepton flavours. As a consequence, ratios of decay rates such as

$$
R_{K}=\frac{\mathscr{B}\left(B^{+} \rightarrow K^{+} \mu^{+} \mu^{-}\right)}{\mathscr{B}\left(B^{+} \rightarrow K^{+} e^{+} e^{-}\right)}
$$

are expected to be very close to unity, save from very small Higgs penguin contributions and difference in phase space due to the lepton mass. The LHCb collaboration has measured $R_{K}$ in the $1<q^{2}<6 \mathrm{GeV}^{2} / c^{4}$ range to be $0.745_{-0.074}^{+0.090}$ (stat) \pm 0.036 (syst) [45], $2.6 \sigma$ away from the SM prediction of $R_{K}=1.0003 \pm 0.0001$ [46]. While the significance of this discrepancy is not enough to be considered even as evidence, the combined $4.0 \sigma$ enhancement, shown in Fig. 14, of $\tau$ with respect to $\mu$ in tree-level $B^{+} \rightarrow D^{(*)} \ell^{+} v_{\ell}$ decays observed by BaBar, Belle and LHCb [47], has prompted great theoretical interest in these types of measurements.

Interpretation While the results discussed so far are basically compatible with the SM picture, a pattern of NP seems to start emerging in flavour physics, with two different sets of anomalies in $b \rightarrow s$ transitions: angular distributions of $b \rightarrow s \ell^{+} \ell^{-}$decays and lepton flavour universality violation. These point to a preference for sizable NP in vector leptonic couples and lepton non-universality, leaving room for the contribution of non-SM right-handed currents and, thus, a non-MFV flavour sector. 

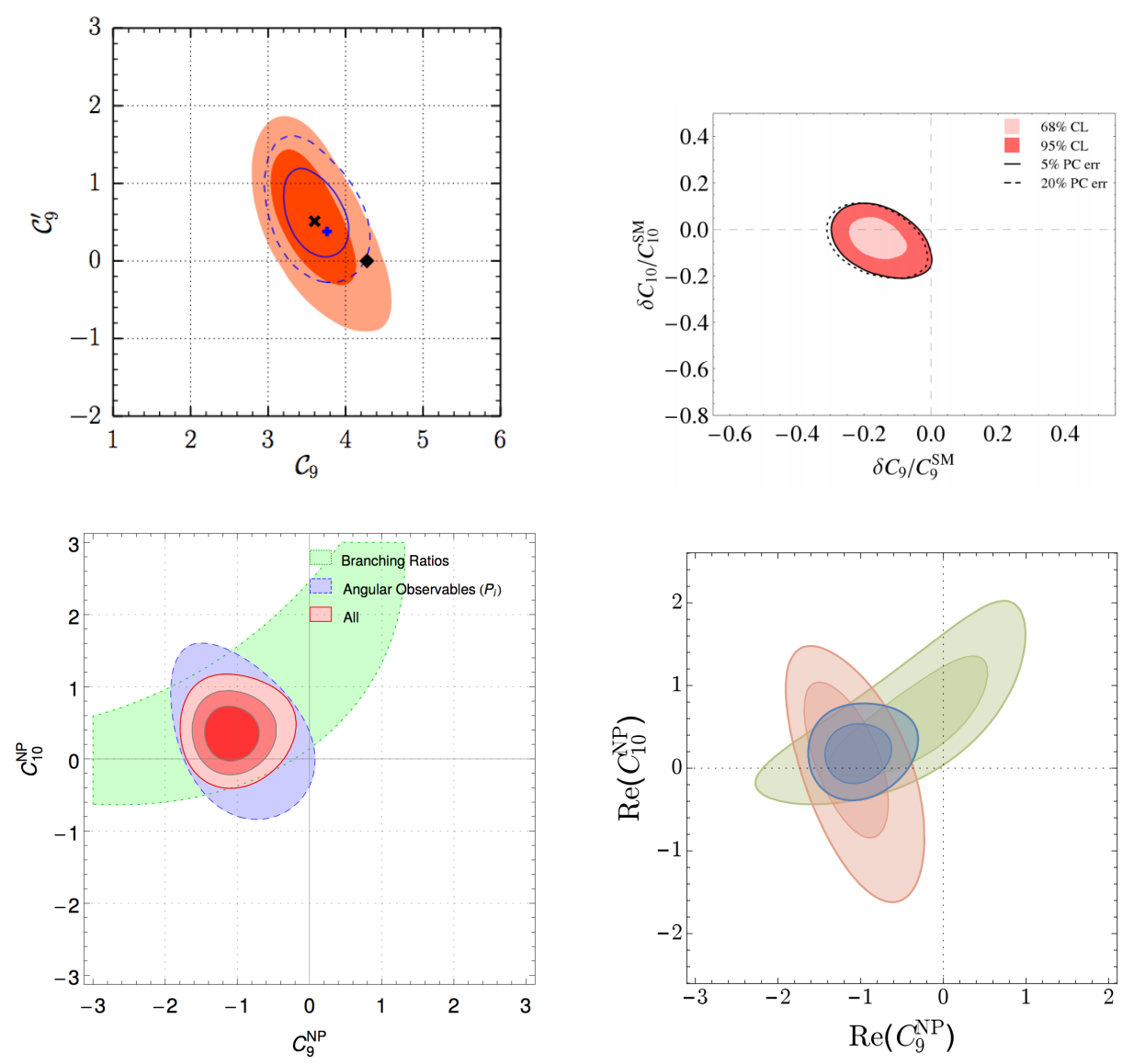

Figure 13: Results from the global fits to $b \rightarrow s$ observables: the top left plot, from Ref. [41], shows the $C_{9}-C_{9}^{\prime}$ plane, including NP contributions, with the SM prediction represented as a black dot; on the top right, taken from Ref. [42] one can see the one- and two-sigma contours of the relative NP contributions in $C_{9}$ and $C_{10}$, with the SM being at $(0,0)$; the bottom left plot, from Ref. [43], shows, in red, directly the contours of the value of the NP contributions in $C_{9}$ and $C_{10}$, highlighting as well the contributions coming only from angular observables (blue) and branching fractions (green); the bottom right plot, taken from Ref. [44], shows, in blue, the contours of the real values of the NP contributions in $C_{9}$ and $C_{10}$, also highlighting the contributions coming only from angular observables (red) and branching fractions (green).

Several types of models have been built to explain these hints of anomalies, including the existence $Z^{\prime}$ bosons [54,55] or leptoquarks [56,57], as well as the gauged $L_{\tau}-L_{\mu} \mathrm{SM}$ extension [58, 59]. There exists, however, concern in the theory community about to which extent the longdistance contributions from $c \bar{c}$ resonances pollute the observables and how factorisation holds in this case [60]; it is hard to answer those concerns from first principles, so it is necessary to use models to try to measure the size of the $c \bar{c}$ pollution. While this could affect the uncertainty of the SM prediction in the case of angular observables, reducing the significance of the anomalies, measurements such as $R_{K}$ are mostly free of this type of hadronic uncertainties. 


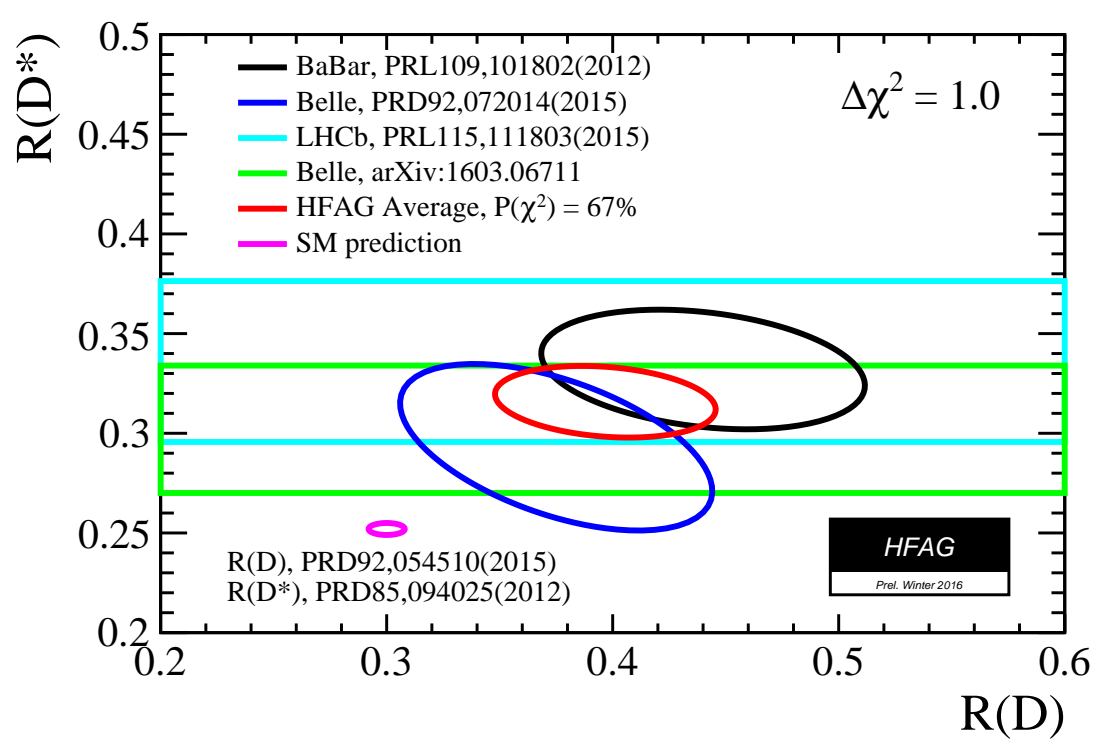

Figure 14: One sigma contour (red) of the combination of the BaBar (black) [48], Belle (green and dark blue) [49,50] and LHCb (cyan) [51] results of the ratio of tauonic and muonic channels in $B^{+} \rightarrow D \ell^{+} v_{\ell}$ and $B^{+} \rightarrow D^{*} \ell^{+} v_{\ell}$ decays, taken from Ref. [47], with the SM prediction from Refs. [52,53] overlaid in magenta.

\section{Other rare decays}

Rare charm decays The short-distance contributions to rare $c \rightarrow u$ transitions are very small due to the stronger GIM suppression $\left(m_{b}<<m_{t}\right)$, so rare charm decays are dominated by long-distance contributions.

While the current state of measurements, shown in Fig. 15, is still not close to the SM predictions, typically of $<\mathscr{O}\left(10^{-9}\right)$, large improvements on the limits have been achieved at the LHC. In particular, LHCb has significantly improved the limits in the searches for the FCNC $D^{0} \rightarrow \pi^{+} \pi^{-} \mu^{+} \mu^{-}$[61], $D^{0} \rightarrow \mu^{+} \mu^{-}$[62] and $D_{(s)}^{+} \rightarrow \pi^{+} \mu^{+} \mu^{-}$[63] decays, and the LFV $D^{0} \rightarrow e^{ \pm} \mu^{\mp}$ decay [64]. Additionally, it has performed the first observation of the $D^{0} \rightarrow K^{-} \pi^{+} \mu^{+} \mu^{-}$decay in the $\rho$ - $\omega$ region in $\mu^{+} \mu^{-}$mass [65], necessary to understand the long-distance, tree-level contributions of vector resonances to the rare $D \rightarrow X \mu^{+} \mu^{-}$mode.

Great improvements are expected in the Run II of the LHC thanks to the improved trigger strategy at $\mathrm{LHCb}$ - increasing the sample size by more than proportionally to the luminosity — with the potential for reaching even more interesting regions in the LHCb upgrade.

Rare strange decays While the LHC is not the main player in rare kaon physics, a very competitive result in the search for the $K_{\mathrm{s}}^{0} \rightarrow \mu^{+} \mu^{-}$decay was published by the LHCb collaboration using data from 2011 [66]. The limit on the branching fraction of this decay,

$$
\mathscr{B}\left(K_{\mathrm{S}}^{0} \rightarrow \mu^{+} \mu^{-}\right)<9 \times 10^{-9} \text { at } 90 \% \text { C.L. }
$$

is still far from the SM prediction of $\mathscr{B}\left(K_{\mathrm{S}}^{0} \rightarrow \mu^{+} \mu^{-}\right)=(5.1 \pm 1.5) \times 10^{-12}$ [67], but the LHC has the potential of reaching the most interesting region of study, in which it will be possible to assess possible NP short-distance effects in the $K_{\mathrm{L}}^{0} \rightarrow \mu^{+} \mu^{-}$decay. 

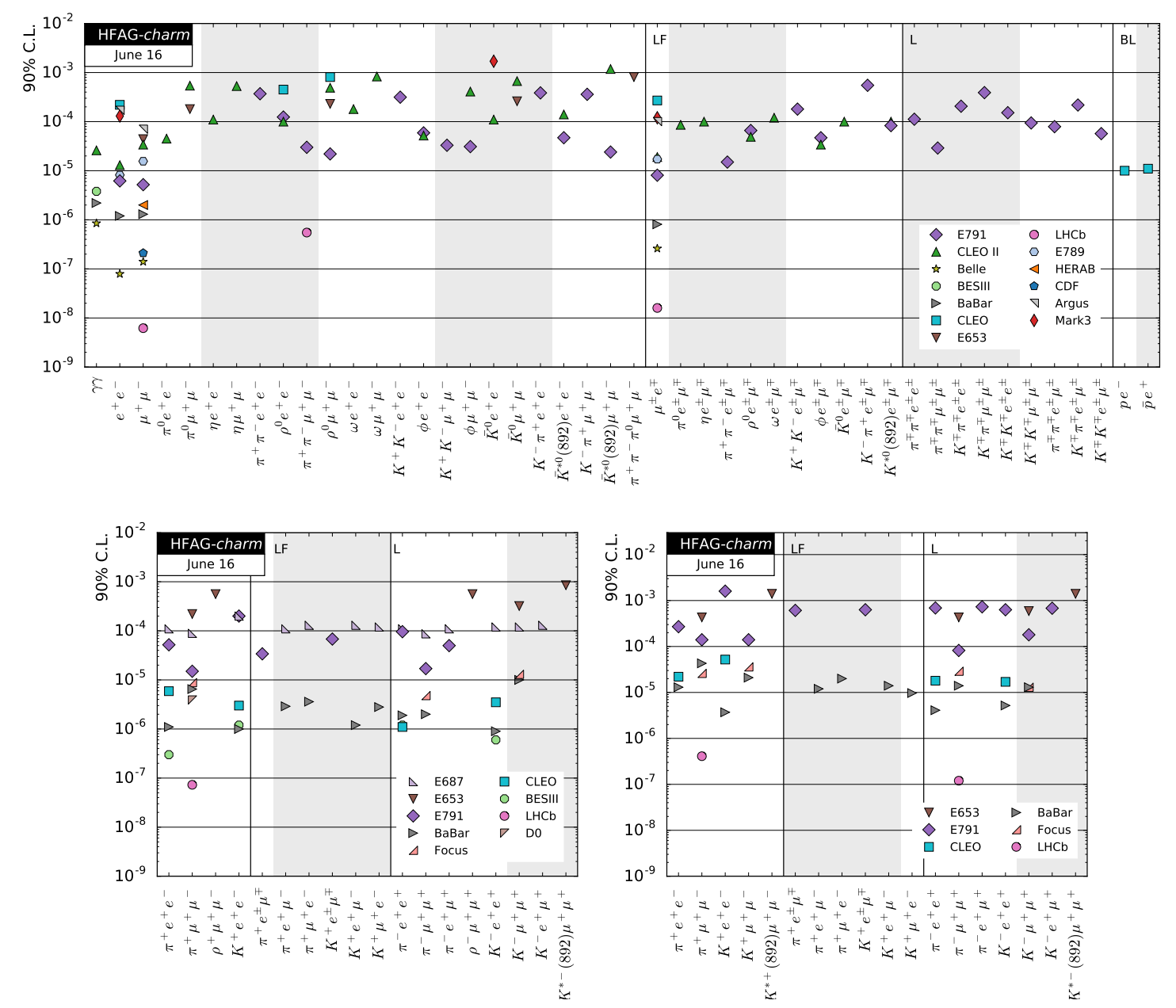

Figure 15: Status of rare charm searches for $D^{0}$ (top), $D^{+}$(bottom left) and $D_{s}^{+}$(bottom right) mesons [47].

The limit $K_{\mathrm{S}}^{0} \rightarrow \mu^{+} \mu^{-}$on shows, nonetheless, the potential of LHCb to produce significant results in the following years—especially in its Upgrade phase-such as the study of the $\Sigma^{+} \rightarrow$ $p \mu^{+} \mu^{-}$decay to assess the HyperCP anomaly [68], the update of the limit $K_{\mathrm{S}}^{0} \rightarrow \mu^{+} \mu^{-}$with the full Run I dataset, and the exploration of further modes, including those with electrons in their final state. These all will contribute to the exciting prospects from non-LHC experiments: NA62 took its first data in 2015 and is currently getting ready for its 2016 run, while KOTO is expecting to reach SM sensitivity in the search for the $K_{\mathrm{L}}^{0} \rightarrow \pi^{0} v v$ decay by 2018 .

Hidden sector The $b \rightarrow s$ penguin decay is also an excellent place to search for low-mass hidden sector particles, which can mix with the Higgs boson and then decay in SM final states. The LHCb collaboration has performed a search for hidden-sector bosons in $B^{0} \rightarrow K^{* 0} \chi\left(\rightarrow \mu^{+} \mu^{-}\right)$decays allowing — but not requiring — non zero lifetime of the $\mu^{+} \mu^{-}$system [69]. The search at different lifetimes, covering prompt and displaced $\mu^{+} \mu^{-}$vertices and shown in Fig.16, found no significant signal and allowed both to set model-independent limits and to constrain specific models, such as the ones described in Refs. [70-73]. 


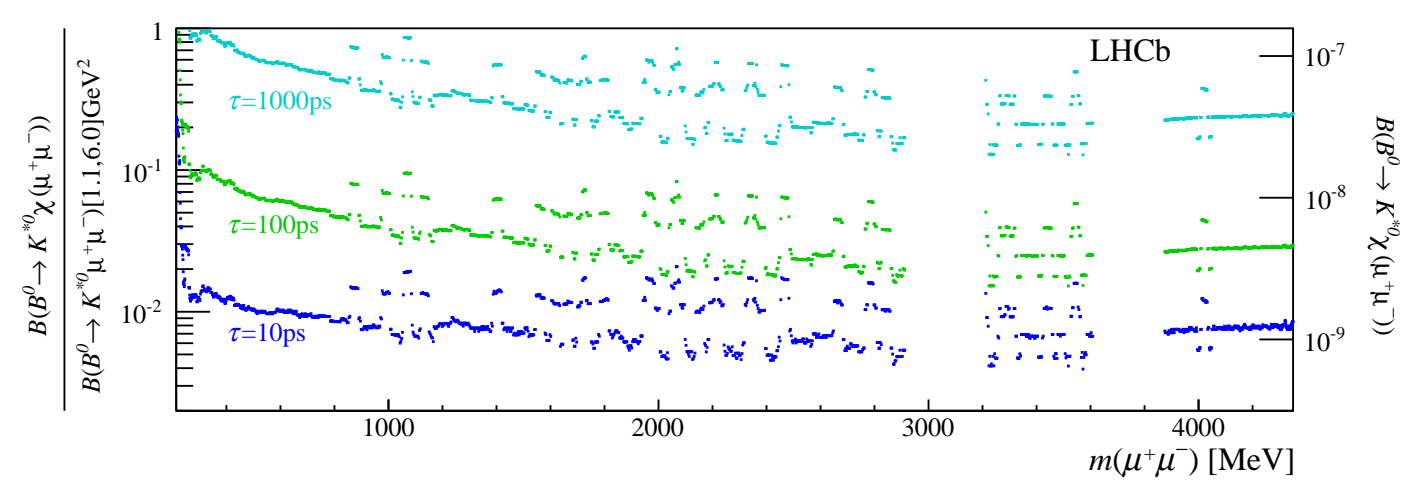

Figure 16: Upper limit at $95 \%$ C.L. for the $B^{0} \rightarrow K^{* 0} \chi\left(\rightarrow \mu^{+} \mu^{-}\right)$decay as a function of the $\mu^{+} \mu^{-}$mass, obtained at different lifetimes of the $\mu^{+} \mu^{-}$system [69].

\section{Conclusions}

Rare flavoured decay observables place strong constraints on many NP models, allowing to probe higher energies than direct searches due to the fact that they are forbidden at tree-level in the SM. A large number of analyses performed using data collected during Run I of the LHC have lead to substantial improvement in the precision of several key observables. Results like the observation of the $B_{s}^{0} \rightarrow \mu^{+} \mu^{-}$decay or the study of angular observables in $B^{0} \rightarrow K^{* 0} \mu^{+} \mu^{-}$, while largely consistent with the SM, have given rise to interesting tensions with the SM expectations.

While there is not significant NP evidence from a single measurement, global fits to rare decays observables point to a pattern that favours the existence of NP. In this situation, it is necessary to continue improving the precision and to add measurements, both of new, more sensitive observables, and of new decay modes. An effort in the theory side, especially in the reduction of uncertainties due to hadronic effects, will also be needed.

In a nutshell, the main goal in the next few years will be to try to confirm these tensions of the SM, find the first evidences of NP and then study their features to determine which models are favoured by the data.

\section{References}

[1] S. L. Glashow, J. Iliopoulos and L. Maiani, Weak Interactions with Lepton-Hadron Symmetry, Phys. Rev. D2 (Oct, 1970) 1285-1292.

[2] N. Cabibbo, Unitary Symmetry and Leptonic Decays, Phys. Rev. Lett. 10 (Jun, 1963) 531-533.

[3] M. Kobayashi and T. Maskawa, CP-Violation in the Renormalizable Theory of Weak Interaction, Prog. Theor. Phys. 49 (1973) 652-657.

[4] K. G. Wilson and W. Zimmermann, Operator product expansions and composite field operators in the general framework of quantum field theory, Commun. Math. Phys. 24 (1972) 87-106.

[5] G. Buchalla, A. J. Buras and M. E. Lautenbacher, Weak decays beyond leading logarithms, Rev. Mod. Phys. 68 (1996) 1125-1144, [hep-ph/9512380].

[6] K. G. Chetyrkin, M. Misiak and M. Munz, Weak radiative B meson decay beyond leading logarithms, Phys. Lett. B400 (1997) 206-219, [hep-ph/ 9612313$].$ 
[7] C. Bobeth, M. Gorbahn, T. Hermann, M. Misiak, E. Stamou and M. Steinhauser, $B_{s, d} \rightarrow l^{+} l^{-}$in the Standard Model with Reduced Theoretical Uncertainty, Phys. Rev. Lett. 112 (2014) 101801, [1311.0903].

[8] D. M. Straub, New physics correlations in rare decays, in CKM unitarity triangle. Proceedings, 6th International Workshop, CKM 2010, Warwick, UK, September 6-10, 2010. 1012.3893.

[9] CLEO collaboration, P. Avery et al., Upper Limit on Flavor Changing Neutral Current Decays of the $b$ Quark, Phys. Rev. Lett. 53 (1984) 1309-1313.

[10] CMS AND LHCB collaboration, V. Khachatryan et al., Observation of the rare $B_{s}^{0} \rightarrow \mu^{+} \mu^{-}$decay from the combined analysis of CMS and LHCb data, Nature 522 (2015) 68 CERN-PH-EP-2014-220, CMS-BPH-13-007, LHCb-PAPER-2014-049, [1411 . 4413].

[11] D. M. Straub, New Physics Searches in Flavour Physics, Nuovo Cim. C035N1 (2012) 249-256, [1107.0266].

[12] ATLAS collaboration, M. Aaboud et al., Study of the rare decays of $B_{s}^{0}$ and $B^{0}$ into muon pairs from data collected during the LHC Run 1 with the ATLAS detector, 1604.04263.

[13] LHCв collaboration, R. Aaij et al., Differential branching fractions and isospin asymmetries of $B \rightarrow K^{(*)} \mu^{+} \mu^{-}$decays, JHEP 06 (2014) 133 LHCb-PAPER-2014-006, CERN-PH-EP-2014-055, [1403.8044].

[14] LHCB collaboration, R. Aaij et al., Measurement of the $S$-wave fraction in $B^{0} \rightarrow K^{+} \pi^{-} \mu^{+} \mu^{-}$decays and the $B^{0} \rightarrow K^{*}(892)^{0} \mu^{+} \mu^{-}$differential branching fraction, 1606.04731

LHCb-PAPER-2016-012, CERN-EP-2016-141, [1606.04731].

[15] CMS collaboration, S. Chatrchyan et al., Angular analysis and branching fraction measurement of the decay $B^{0} \rightarrow K^{* 0} \mu^{+} \mu^{-}$, Phys. Lett. B727 (2013) 77-100, [1308. 3409 ].

[16] CMS collaboration, V. Khachatryan et al., Angular analysis of the decay $B^{0} \rightarrow K^{* 0} \mu^{+} \mu^{-}$from pp collisions at $\sqrt{s}=8 \mathrm{TeV}$, Phys. Lett. B753 (2016) 424-448, [1507.08126].

[17] LHCB collaboration, R. Aaij et al., Angular analysis and differential branching fraction of the decay $B_{s}^{0} \rightarrow \phi \mu^{+} \mu^{-}$, JHEP 09 (2015) 179 LHCb-PAPER-2015-023, CERN-PH-EP-2015-145, [1506.08777].

[18] LHCB collaboration, R. Aaij et al., Differential branching fraction and angular anaysis of $\Lambda_{b}^{0} \rightarrow \Lambda \mu^{+} \mu^{-}$decays, JHEP 06 (2015) 115 LHCb-PAPER-2015-009, CERN-PH-EP-2015-078, [1503.07138].

[19] C. Bobeth, G. Hiller and D. van Dyk, More benefits of semileptonic rare B decays at low recoil: $C P$ Violation, JHEP 07 (2011) 067, [1105.0376].

[20] HPQCD collaboration, C. Bouchard, G. P. Lepage, C. Monahan, H. Na and J. Shigemitsu, Rare decay $B \rightarrow K \ell^{+} \ell^{-}$form factors from lattice QCD, Phys. Rev. D88 (2013) 054509, [1306.2384].

[21] R. R. Horgan, Z. Liu, S. Meinel and M. Wingate, Calculation of $B^{0} \rightarrow K^{* 0} \mu^{+} \mu^{-}$and $B_{s}^{0} \rightarrow \phi \mu^{+} \mu^{-}$ observables using form factors from lattice QCD, Phys. Rev. Lett. 112 (2014) 212003, [1310 . 3887 ].

[22] W. Altmannshofer and D. M. Straub, New physics in $b \rightarrow$ s transitions after LHC run 1, Eur. Phys. J. C75 (2015) 382, [1411.3161].

[23] A. Bharucha, D. M. Straub and R. Zwicky, $B \rightarrow V \ell^{+} \ell^{-}$in the Standard Model from light-cone sum rules, JHEP 08 (2016) 098, [1503.05534]. 
[24] W. Detmold and S. Meinel, $\Lambda_{b} \rightarrow \Lambda \ell^{+} \ell^{-}$form factors, differential branching fraction, and angular observables from lattice QCD with relativistic b quarks, Phys. Rev. D93 (2016) 074501, [1602.01399].

[25] R. R. Horgan, Z. Liu, S. Meinel and M. Wingate, Lattice QCD calculation of form factors describing the rare decays $B \rightarrow K^{*} \ell^{+} \ell^{-}$and $B_{s} \rightarrow \phi \ell^{+} \ell^{-}$, Phys. Rev. D89 (2014) 094501, [1310 . 3722].

[26] LHCB collaboration, R. Aaij et al., First measurement of the differential branching fraction and CP asymmetry of the $B^{+} \rightarrow \pi^{+} \mu^{+} \mu^{-}$decay, JHEP 10 (2015) 034 LHCb-PAPER-2015-035, CERN-PH-EP-2015-219, [1509.00414].

[27] A. Ali, A. Ya. Parkhomenko and A. V. Rusov, Precise Calculation of the Dilepton Invariant-Mass Spectrum and the Decay Rate in $B^{ \pm} \rightarrow \pi^{ \pm} \mu^{+} \mu^{-}$in the SM, Phys. Rev. D89 (2014) 094021, [1312.2523].

[28] C. Hambrock, A. Khodjamirian and A. Rusov, Hadronic effects and observables in $B \rightarrow \pi \ell^{+} \ell^{-}$decay at large recoil, Phys. Rev. D92 (2015) 074020, [1506.07760].

[29] Fermilab Lattice, MILC collaboration, J. A. Bailey et al., $B \rightarrow \pi \ell \ell$ form factors for new-physics searches from lattice QCD, Phys. Rev. Lett. 115 (2015) 152002, [1507.01618].

[30] S. Descotes-Genon, T. Hurth, J. Matias and J. Virto, Optimizing the basis of $B->K^{*}$ ll observables in the full kinematic range, JHEP 05 (2013) 137, [1303 . 5794].

[31] CDF collaboration, T. Aaltonen et al., Measurements of the Angular Distributions in the Decays $B \rightarrow K^{(*)} \mu^{+} \mu^{-}$at CDF, Phys. Rev. Lett. 108 (2012) 081807, [1108. 0695].

[32] BABAR collaboration, J. P. Lees et al., Measurement of angular asymmetries in the decays $B \rightarrow K^{*} \ell^{+} \ell^{-}$, Phys. Rev. D93 (2016) 052015, [1508.07960].

[33] BeLle collaboration, J. T. Wei et al., Measurement of the Differential Branching Fraction and Forward-Backward Asymmetry for $B \rightarrow K^{(*)} \ell^{+} \ell^{-}$, Phys. Rev. Lett. 103 (2009) 171801, [0 904.0770 ].

[34] LHCB collaboration, R. Aaij et al., Angular analysis of the $B^{0} \rightarrow K^{* 0} \mu^{+} \mu^{-}$decay using $3 f b^{-1}$ of integrated luminosity, JHEP 02 (2016) 104 LHCb-PAPER-2015-051, [1512 . 04442 ].

[35] Belle collaboration, A. Abdesselam et al., Angular analysis of $B^{0} \rightarrow K^{*}(892)^{0} \ell^{+} \ell^{-}$, in Proceedings, LHCSki 2016 - A First Discussion of 13 TeV Results: Obergurgl, Austria, April 10-15, 2016, 2016. 1604.04042 .

[36] S. Descotes-Genon, L. Hofer, J. Matias and J. Virto, On the impact of power corrections in the prediction of $B \rightarrow K^{*} \mu^{+} \mu^{-}$observables, JHEP 12 (2014) 125, [1407.8526].

[37] S. Meinel, Flavor physics with $\Lambda_{b}$ baryons, PoS LATTICE2013 (2014) 024, [1401.2685].

[38] LHCB collaboration, R. Aaij et al., Angular analysis of the $B^{0} \rightarrow K^{* 0} e^{+} e^{-}$decay in the low- $q^{2}$ region, JHEP 04 (2015) 064 LHCb-PAPER-2014-066, CERN-PH-EP-2014-301, [1501. 03038].

[39] D. Becirevic and E. Schneider, On transverse asymmetries in $B \rightarrow K^{*} \ell^{+} \ell^{-}$, Nucl. Phys. $\mathbf{B 8 5 4}$ (2012) 321-339, [1106.3283].

[40] S. Jäger and J. Martin Camalich, Reassessing the discovery potential of the $B \rightarrow K^{*} \ell^{+} \ell^{-}$decays in the large-recoil region: SM challenges and BSM opportunities, Phys. Rev. D93 (2016) 014028, [1412.3183].

[41] F. Beaujean, C. Bobeth and D. van Dyk, Comprehensive Bayesian analysis of rare (semi)leptonic and radiative B decays, Eur. Phys. J. C74 (2014) 2897, [1310 . 2478]. 
[42] T. Hurth, F. Mahmoudi and S. Neshatpour, On the anomalies in the latest LHCb data, Nucl. Phys. B909 (2016) 737-777, [1603.00865].

[43] S. Descotes-Genon, L. Hofer, J. Matias and J. Virto, Global analysis of $b \rightarrow$ sll anomalies, JHEP 06 (2016) 092, [1510.04239].

[44] W. Altmannshofer and D. M. Straub, Implications of $b \rightarrow s$ measurements, in Proceedings, 50th Rencontres de Moriond Electroweak Interactions and Unified Theories: La Thuile, Italy, March 14-21, 2015, pp. 333-338, 2015. 1503.06199.

[45] LHCв collaboration, R. Aaij et al., Test of lepton universality using $B^{+} \rightarrow K^{+} \ell^{+} \ell^{-}$decays, Phys. Rev. Lett. 113 (2014) 151601 LHCb-PAPER-2014-024, CERN-PH-EP-2014-140, [1 406 . 6482].

[46] C. Bobeth, G. Hiller and G. Piranishvili, Angular distributions of $\bar{B} \rightarrow \bar{K} \ell^{+} \ell^{-}$decays, JHEP 12 (2007) 040, [0 709.4174$]$.

[47] Heavy Flavor Averaging Group collaboration, Y. Amhis et al., Averages of b-hadron, c-hadron, and $\tau$-lepton properties as of summer 2014, 1412.7515.

[48] BABAR collaboration, J. P. Lees et al., Evidence for an excess of $\bar{B} \rightarrow D^{(*)} \tau^{-} \bar{v}_{\tau}$ decays, Phys. Rev. Lett. 109 (2012) 101802, [1205.5442].

[49] BELLE collaboration, M. Huschle et al., Measurement of the branching ratio of $\bar{B} \rightarrow D^{(*)} \tau^{-} \bar{v}_{\tau}$ relative to $\bar{B} \rightarrow D^{(*)} \ell^{-} \bar{v}_{\ell}$ decays with hadronic tagging at Belle, Phys. Rev. D92 (2015) 072014, [1507.03233].

[50] BELLE collaboration, A. Abdesselam et al., Measurement of the branching ratio of $\bar{B}^{0} \rightarrow D^{*+} \tau^{-} \bar{v}_{\tau}$ relative to $\bar{B}^{0} \rightarrow D^{*+} \ell^{-} \bar{v}_{\ell}$ decays with a semileptonic tagging method, 1603.06711.

[51] LHCB collaboration, R. Aaij et al., Measurement of the ratio of branching fractions $\mathscr{B}\left(\bar{B}^{0} \rightarrow D^{*+} \tau^{-} \bar{v}_{\tau}\right) / \mathscr{B}\left(\bar{B}^{0} \rightarrow D^{*+} \mu^{-} \bar{v}_{\mu}\right)$, Phys. Rev. Lett. 115 (2015) 111803

LHCb-PAPER-2015-025, CERN-PH-EP-2015-150, [1506.08614].

[52] HPQCD collaboration, H. Na, C. M. Bouchard, G. P. Lepage, C. Monahan and J. Shigemitsu, $B \rightarrow D \ell v$ form factors at nonzero recoil and extraction of $\left|V_{c b}\right|$, Phys. Rev. D92 (2015) 054510, [1505. 03925].

[53] S. Fajfer, J. F. Kamenik and I. Nisandzic, On the $B \rightarrow D^{*} \tau \bar{v}_{\tau}$ Sensitivity to New Physics, Phys. Rev. D85 (2012) 094025, [1203.2654].

[54] R. Gauld, F. Goertz and U. Haisch, An explicit $Z^{\prime}$-boson explanation of the $B \rightarrow K^{*} \mu^{+} \mu^{-}$anomaly, JHEP 01 (2014) 069, [1310.1082].

[55] A. J. Buras, F. De Fazio and J. Girrbach, 331 models facing new $b \rightarrow s \mu^{+} \mu^{-}$data, JHEP 02 (2014) $112,[1311.6729]$.

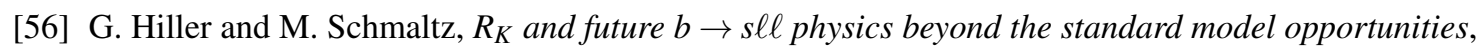
Phys. Rev. D90 (2014) 054014, [1408 .1627].

[57] B. Gripaios, M. Nardecchia and S. A. Renner, Composite leptoquarks and anomalies in B-meson decays, JHEP 05 (2015) 006, [1412.1791].

[58] P. J. Fox, J. Liu, D. Tucker-Smith and N. Weiner, An Effective Z', Phys. Rev. D84 (2011) 115006, [1104.4127].

[59] W. Altmannshofer, S. Gori, M. Pospelov and I. Yavin, Quark flavor transitions in $L_{\mu}-L_{\tau}$ models, Phys. Rev. D89 (2014) 095033, [1403.1269].

[60] J. Lyon and R. Zwicky, Resonances gone topsy turvy - the charm of QCD or new physics in $b \rightarrow s \ell^{+} \ell^{-}$?, 1406.0566 . 
[61] LHCB collaboration, R. Aaij et al., Search for the decay $D^{0} \rightarrow \pi^{+} \pi^{-} \mu^{+} \mu^{-}$, Phys. Lett. B728 (2014) 234 LHCb-PAPER-2013-050, CERN-PH-EP-2013-183, [1310 . 2535].

[62] LHCB collaboration, R. Aaij et al., Search for the rare decay $D^{0} \rightarrow \mu^{+} \mu^{-}$, Phys. Lett. B725 (2013) 15 CERN-PH-EP-2013-083, LHCb-PAPER-2013-013, [1305. 505 9].

[63] LHCB collaboration, R. Aaij et al., Search for $D_{(s)}^{+} \rightarrow \pi^{+} \mu^{+} \mu^{-}$and $D_{(s)}^{+} \rightarrow \pi^{-} \mu^{+} \mu^{+}$decays, Phys. Lett. B724 (2013) 203 LHCb-PAPER-2012-051, CERN-PH-EP-2013-061, [1304 . 6365].

[64] LHCB collaboration, R. Aaij et al., Search for the lepton-flavour violating decay $D^{0} \rightarrow e^{ \pm} \mu^{\mp}$, Phys. Lett. B754 (2016) 167 LHCb-PAPER-2015-048, CERN-PH-EP-2015-306, [1512 . 00322 ].

[65] LHCB collaboration, R. Aaij et al., First observation of the decay $D^{0} \rightarrow K^{-} \pi^{+} \mu^{+} \mu^{-}$in the $\rho^{0}-\omega$ region of the dimuon mass spectrum, Phys. Lett. B757 (2016) 558 LHCb-PAPER-2015-043, CERN-PH-EP-2015-283, [1510.08367].

[66] LHCB collaboration, R. Aaij et al., Search for the rare decay $K_{S}^{0} \rightarrow \mu^{+} \mu^{-}$, JHEP 01 (2013) 090 CERN-PH-EP-2012-267, LHCb-PAPER-2012-023, [1209. 402 9].

[67] G. Isidori and R. Unterdorfer, On the short distance constraints from $K_{L, S} \rightarrow \mu^{+} \mu^{-}, J H E P ~ 01 ~(2004)$ 009, [hep-ph/0311084].

[68] HyPERCP collaboration, H. Park et al., Evidence for the decay $\Sigma^{+} \rightarrow p \mu^{+} \mu^{-}$, Phys. Rev. Lett. 94 (2005) 021801, [hep-ex/0501014].

[69] LHCB collaboration, R. Aaij et al., Search for hidden-sector bosons in $B^{0} \rightarrow K^{* 0} \mu^{+} \mu^{-}$decays, Phys. Rev. Lett. 115 (2015) 161802 LHCb-PAPER-2015-036, CERN-PH-EP-2015-202, [1508 . 04094 ].

[70] M. Shaposhnikov and I. Tkachev, The nuMSM, inflation, and dark matter, Phys. Lett. B639 (2006) 414-417, [hep-ph/0604236].

[71] A. Anisimov, Y. Bartocci and F. L. Bezrukov, Inflaton mass in the nuMSM inflation, Phys. Lett. B671 (2009) 211-215, [0809.1097].

[72] F. Bezrukov and D. Gorbunov, Light inflaton Hunter's Guide, JHEP 05 (2010) 010, [0 912 . 0390 ].

[73] F. Bezrukov and D. Gorbunov, Relic Gravity Waves and 7 keV Dark Matter from a GeV scale inflaton, Phys. Lett. B736 (2014) 494-498, [1403 . 4638]. 